\title{
Inside an Angel's mind
}

Bare skin brushes

the cold rough pavement

My feet are naked

on the ground

Today is special:

I have been selected

the chosen one, elected

chosen for a duty

to fulfill my purpose.

I see a bridge, nervous

I climb over, ready

on my platform

Someone sees me,

they frown,

shouting, loudly,

'get down now!'

Strangers restrain

Stop this!

You are mistaken

I have been chosen.

A new reality has dawned upon this icy, long night.

Extreme recklessness has left me broke, alone, blunted.

I look back; not able to comprehend no insight.

My mind is challenged, reality confronted.

Believing I could reach heaven, how is that possible?

I had wings, heavy, where do they hide?

Now Drained; I was completely inexhaustible.

Empty inside, I weep, thinking I nearly died.

\section{Charlotte Cliffe}

Correspondence to Dr. Charlotte Cliffe, Junior doctor and MA student, Dickson Poon Law School, Kings College London, UK; charlotte.cliffe@nhs.net

Competing interests None declared.

Provenance and peer review Not commissioned; internally peer reviewed.

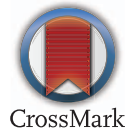

To cite Cliffe C. Med Humanit 2017:43:136.

Published Online First 13 January 2017

Med Humanit 2017:43:136. doi:10.1136/medhum-2016-011128 